\title{
Study on an Emotion Generation Model for a Robot Using a Chaotic Neural Network
}

\author{
Hiroyuki Sumitomo $^{1}$, Masataka Tokumaru ${ }^{2}$, and Noriaki Muranaka ${ }^{2}$ \\ ${ }^{1}$ Graduate School of Kansai University, 3-3-35 Yamate-cho Suita-shi, Osaka, 564-8680 Japan \\ ${ }^{2}$ Kansai University, 3-3-35 Yamate-cho Suita-shi, Osaka, 564-8680 Japan \\ k067993akansai-u.ac.jp
}

\begin{abstract}
This paper proposes an emotion-generation model for complex change using a chaotic neural network $(\mathrm{CNN})$. Using a $\mathrm{CNN}$, the proposed model will solve the problem of past studies that have indicated that robotic emotion changes are simplistic. The model uses the principle of an adaptation level, which is used in Russell's emotion model to generate emotion. This paper considers the effectiveness of this approach using simulation, and shows that the model can express a change of "adaptation". In addition, through the chaos of $\mathrm{CNN}$, the proposed model can express different changes, even if the values of CNN's input values remain the same.
\end{abstract}

Keywords: Partner Robot, Chaos Neural Network, Emotion.

\section{Introduction}

Recently, the market for partner robot has expanded with along with technological advances. A partner robot is a robot that receives communications from a person and brings healing to the person's mind. However, because the expressions of emotion that it brings are simple, both interest and research studies on this subject have waned. This study examines, using a chaotic neural network (CNN), a model that can generate complex emotions that are similar to the emotions of human beings.

\section{The Emotion-Generation Model}

The emotion-dimension theory is used to construct the emotions of a partner robot. In this paper, Russell's emotion model can express emotion by two dimensions consisting of two axes, the pleasant level and the awake level [1]. Russell's emotion model also exhibits the principle of adaptation. It shows that an emotion is effected by the emotion of the previous event. For example, a glad event when a emotion is a little sad is felt gladder than usually. In this paper, CNN is used to show a complex output change [2]. This is followed by the details of the emotiongeneration model, and the network structure of $\mathrm{CNN}$, which uses a layered network. 


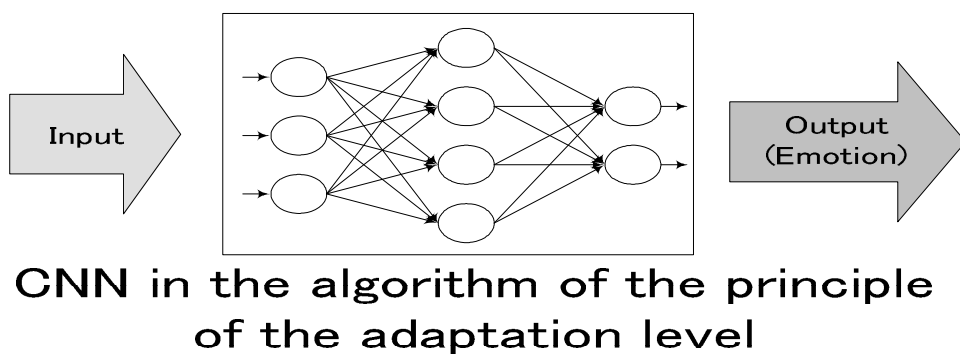

Fig. 1. Structure of the proposed model

$$
\begin{gathered}
y(t)=\eta(t)+\zeta(t) \\
x(t)-0.42 x(t-1)=\frac{2}{1+\exp (-(\eta(t)+\zeta(t)+\beta(t)) / \varepsilon)}-1 \\
s=1+\exp (-(\eta(t)+\zeta(t)) / \varepsilon) \\
y(t)=-\varepsilon \log \left\{\frac{s}{1-0.21 s x(t-1)}-1\right\}
\end{gathered}
$$

In addition, the study method uses a back-propagation algorithm. A structural drawing of the proposed model is shown in Figure 1. To express complex affective changes, the proposed model includes an algorithm that exhibits the principle of the CNN adaptation level. The expression in an internal state of the neuron of CNN is (1). For the proposed model, adding the principle of adaptation level to (1), (1) changes (3) and (4), using (2).

The proposed model prepares five inputs. Two of them take either -1 or 1 at a constant probability, and neither values becomes 1 . Another two inputs change the probability that the value is -1 or 1 , according to other inputs. These four values do not change for a period that is decided at random. The remaining input takes the value of two significant figures for -1 to 1 . In addition all inputs take a gradual time-series change.

\section{Simulation}

In this simulation, the proposed model shows the feature of the adaptation level, and we confirm whether the changes in output of the proposed model are more complex than that of CNN. At first, both models study enough with a same input value.

Next, the output changes of both models were compared when they have the same input value. The results of five trials are shown in Figure 2 and 3. 


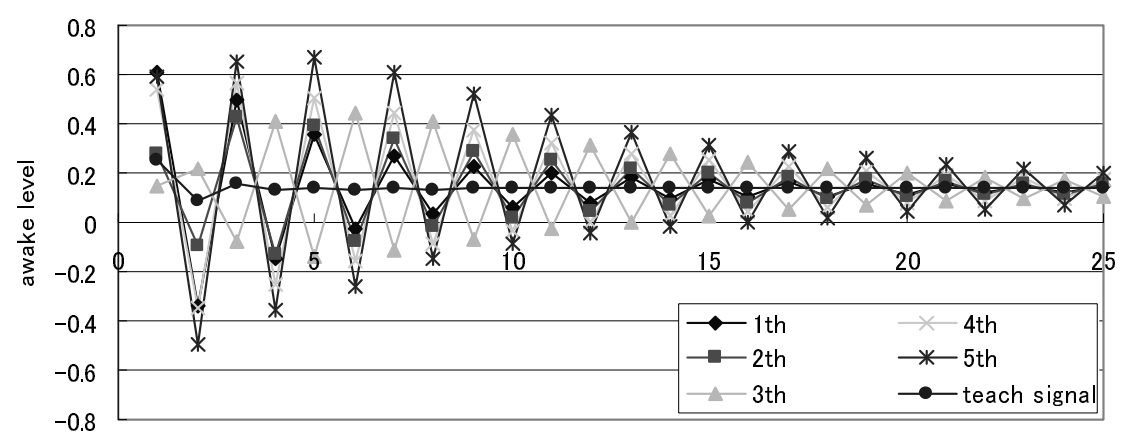

the number of times

Fig. 2. Output change of the proposed model

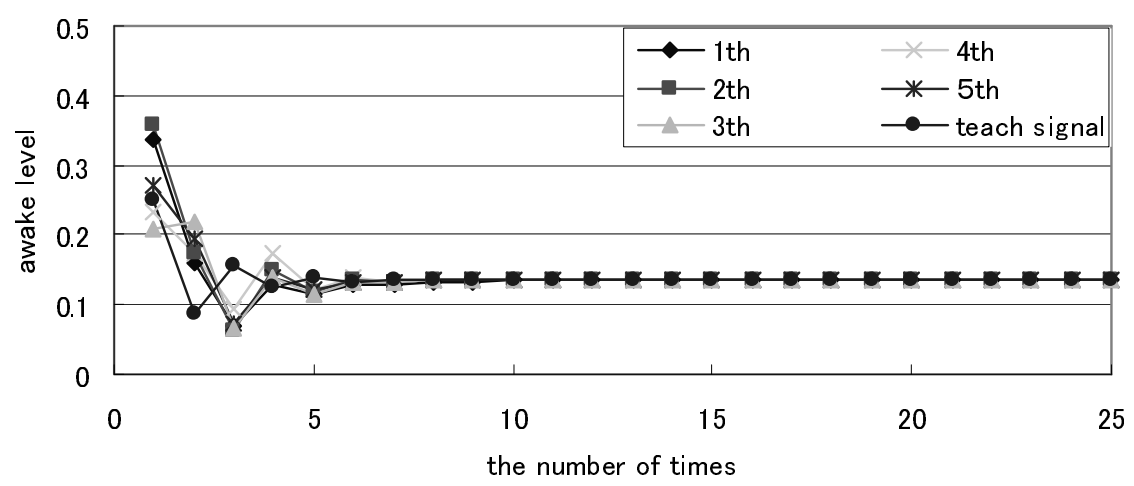

Fig. 3. Output change of CNN

Figure 2 and 3 show an awake level's changes in Russell's emotion-generation model.

For the proposed model, the output change increases and decreases alternately as the value receives the influence the principle of the adaptation level. However, in $\mathrm{CNN}$, it was almost steady with the value of the teach signal.

Moreover, the proposed model can clearly confirm how the output can be settled differently at each trial, although the chaos generated because of the initial weight values of neurons are different. Therefore, we can confirm that the proposed model shows more complex changes of emotion than CNN.

I will evaluate an emotion of the robot with the proposed model.

\section{References}

1. Hama, H., Suzuki, N., Hama, Y.: Invitation to emotion psychology. In: Approach to feelings and emotion, Saiensu-Sya Ltd.

2. Gouhara, K.: Chaos of neural system. Tokyo Denki University Publication 